JOÃO PAULO SANTOS LONGO

\title{
O ENSINO REMOTO NO BRASIL EM TEMPOS DE PANDEMIA:
}

DIÁLOGOS ACERCA DA QUALIDADE E DO DIREITO E ACESSO À EDUCAÇÃO.

Ituiutaba - MG 
JOÃO PAULO SANTOS LONGO

O ENSINO REMOTO NO BRASIL EM TEMPOS DE PANDEMIA:

DIÁLOGOS ACERCA DA QUALIDADE E DO DIREITO E ACESSO À EDUCAÇÃO.

Ituiutaba - MG

2021 


\title{
RESUMO
}

Este artigo, tem como objetivo apresentar os desafios do emprego da tecnologia no ensino remoto e como a situação atual contribuiu para o agravamento da desigualdade. Foi utilizado o método de revisão bibliográfica com abordagem qualitativa de natureza básica. Apresentou-se a relação da tecnologia e a educação, os dispositivos e aplicativos utilizados para fins acadêmicos, aspectos positivos como a troca de informações, mobilidade e dinâmica entre professor e aluno, e questões acerca do uso excessivo da tecnologia, como aspecto negativo. Entendeu-se que o cenário pandêmico, torna-se um agravante da desigualdade social, uma vez que as ferramentas tecnológicas de acesso à internet são impeditivos para alguns. Em conclusão verificou-se que os desafios na implementação das inovações tecnológicas na área da educação básica são extensos e necessários para obtenção de resultados positivos no uso da tecnologia na educação.

PALAVRAS-CHAVE: Tecnologia; Educação; Ferramentas Tecnológicas; Desigualdade.

\begin{abstract}
This article aims to present the challenges of using technology in remote learning and how the current situation has contributed to the aggravation of inequality. The bibliographic review method with a basic qualitative approach was used. The relationship between technology and education, devices and applications used for academic purposes, positive aspects such as the exchange of information, mobility and dynamics between teacher and student, and issues about the excessive use of technology, as a negative aspect, were presented. It was understood that the pandemic scenario becomes an aggravating factor for social inequality, since technological tools and internet access are impediments for some. In conclusion, it was found that the challenges in implementing technological innovations in the area of basic education are extensive and necessary to obtain positive results in the use of technology in education.
\end{abstract}


KEYWORDS: Technology; Education; Technological Tools; Inequality.

\section{- INTRODUÇÃo}

Em dezembro de 2019, na cidade chinesa de Wuhan, apareceram os primeiros casos de uma nova doença, denominada como Coronavírus (COVID-19). Desde então, a população local enfrenta uma crise sanitária global. Após a China ter notificado o surto de casos à OMS (Organização Mundial de Saúde), foram tomadas algumas medidas para conter o avanço da doença no país asiático.

No ano seguinte 2020, a doença já tinha se espalhado por diversos países, fazendo com que a população global adotasse algumas medidas de proteção e prevenção para controlar o avanço do vírus. Dentre as principais medidas, destacase o distanciamento social, que de acordo com Aquino et al. (2020), são "[...] medidas que têm como objetivo reduzir as interações em uma comunidade, que pode incluir pessoas infectadas, ainda não identificadas e, portanto, não isoladas".

Como resultado da medida de distanciamento social, Costa e Nascimento (2020), explicam que as instituições educacionais fecharam suas portas e para dar sequência ao calendário escolar, as atividades continuaram por meio do ensino remoto.

O Ministério da Educação, com a portaria no 343 de 17 de março de 2020 regulamentou a substituição das aulas presenciais por aulas remotas, isto é, aulas mediadas pelas tecnologias, no cenário de pandemia, foram autorizadas em todo 0 território nacional.

Apesar da tecnologia permitir que o calendário escolar seja continuado e que as atividades escolares ocorram sem paralisação, o cenário atual da educação no país escancarou a desigualdade na área da educação. Diante disso, o presente artigo, objetiva apresentar os desafios na utilização da tecnologia no ensino remoto e de como a situação atual contribuiu para o agravamento da desigualdade. 


\section{- FUNDAMENTAÇÃO TEÓRICA}

\section{- TECNOLOGIA}

Constantemente as pessoas são noticiadas sobre o surgimento de uma determinada inovação tecnológica e suas aplicações. Delimitada, por Barreto (1995, p.05), como o "processo de produção de conhecimento", a inovação necessita de uma

zona favorável para que se torne uma realidade. As inovações possuem o poder de atuarem como agentes de mudança na sociedade, uma vez que transformam as vidas tanto dos indivíduos isoladamente, como consequentemente do meio social em que 0 indivíduo está inserido.

Ao longo dos séculos, diversas áreas da sociedade obtiveram benefícios proporcionados pelas evoluções das inovações tecnológicas. Entre as áreas da sociedade, o presente trabalho limitou-se a análise dos desafios na utilização das inovações tecnológicas na área da educação.

A definição de educação envolve diferentes concepções. O conceito filosófico apresentado por Brandão (2007) encara a educação com subjetividade, ressaltando que não basta apenas considerar as condições externas à pessoa na condição de educando, mas também olhar para o que ocorre no seu interior. Já, Araújo et al. (2017, p.925) em um conceito generalista defende que "a educação é um processo, não um fim em si mesmo, portanto precisa sofrer intervenções positivas para o seu aprimoramento". Acatadas estas definições, compreende-se a importância que a educação tem para a sociedade, uma vez que ela atua como agente transformador da sociedade como um todo.

\section{- IMPLEMENTAÇÃO DA TECNOLOGIA NA EDUCAÇÃO}

Hoje, o ambiente de aprendizagem formal não está mais limitado aos muros da escola. A utilização de ferramentas, não disponíveis há algumas décadas, promove 
uma ruptura nos modelos tradicionais de ensino aprendizagem. Este novo cenário exige uma postura crítica acerca da relação entre educação e tecnologia. (MOTA, 2014).

Para Kaieski, Grings e Fetter (2015), as instituições de ensino devem desenvolver programas de aprendizagem voltados para a exploração das tecnologias móveis. Além de obter uma comunicação mais eficaz, o custo da maioria dessas ferramentas é praticamente zero. Claro que a adequação do uso da tecnologia na educação básica não pode ser resumida a um processo simples:

[..] é possível estimular algum nível, ainda que bem introdutório, de contato anterior ilustrativo ao conteúdo. Além do desenvolvimento da prática que estimula e força a autoaprendizagem até os seus limites superiores, a competência digital, que é muito próxima dos jovens, faz parte das habilidades formadoras de um profissional futuro apto a enfrentar os desafios

de um cenário em que a inovação é a marca mais notável (MOTA, 2014).

Nos próximos anos é muito provável que o uso de tecnologias móveis cresça exponencialmente na educação, pois facilita a troca rápida de informações desde um aviso que um professor precise dar aos seus alunos sobre uma aula do dia seguinte até o compartilhamento de aprendizados obtidos em sala de aula. (ALENCAR et al, 2015).

De acordo com Moura (2011) os estudantes utilizam dispositivos e aplicativos mobile em ambientes educacionais basicamente como ferramentas para comunicação, entretenimento e distração própria, ou seja, nem sempre o uso dessas tecnologias está espontaneamente relacionado às atividades de aprendizagem.

Aplicativos tecnológicos são pequenos softwares desenvolvidos especificamente para funcionarem em sistemas operacionais de dispositivos móveis, como celulares ou tablets, por exemplo, sendo que entre suas principais funcionalidades está a possibilidade de interação e navegação com seus usuários, de maneira fácil e ágil. (AMORIN; BIANCO, 2011).

Alguns aplicativos podem ser destacados pela possibilidade do seu uso para fins acadêmicos. No mundo digital, torna-se interessante explorar o tempo em que os 
estudantes passam em internet para promover o desenvolvimento do senso crítico por meio de debates orientados pelo professor. (PECHI, 2013).

O uso pedagógico do Facebook auxilia na cooperação entre os estudantes, promovendo ainda o pensamento crítico e reflexivo. Já o WhatsApp é uma ferramenta de trocas de mensagens que possibilita o envio de imagens, arquivos, emojis, áudios, entre outros. O aplicativo possibilita que professores promovam debates, produções textuais e até mesmo aulas colaborativas. É interessante ressaltar como a ferramenta auxilia na troca de informações entre os alunos, tornando muito mais fácil saber 0 ponto de vista do colega sobre determinado assunto visto em sala de aula. (ALENCAR et al, 2015).

O Moodle é um ambiente virtual de aprendizagem (AVA) que possibilita uma série de atividades que auxiliam na organização do estudante. Entre as utilidades estão: acompanhamento de atividades entregues, visualização de notas, realização de atividades virtuais, entre outros. (BEHAR, 2009).

Martin Dougiamas desenvolveu o Moodle (Modular Object-Oriented Dynamic Learning Environment) em 1999, e a ferramenta é 100\% gratuita. Este software

oferece ao professor uma gama de ferramentas que podem ser utilizadas para interação on-line, facilitando o alcance de seus objetivos pedagógicos. (TORRES; SILVA, 2008).

O Teams é um software da Microsoft desenvolvido para a colaboração de equipes. A concepção inicial do aplicativo foi para colaboração de equipes corporativas, mas recentemente tem sido utilizado inclusive para fins educacionais. Ele reúne conversas, conteúdo e aplicativos em um só lugar, simplificando o fluxo de trabalho dos coordenadores e diretores, simultaneamente, permite que os professores criem ambientes personalizados de aprendizado. (MOODLE CEAD UFU, [s.d.]).

\section{- DESAFIOS DO USO DE FERRAMENTAS TECNOLÓGICAS}


A pesquisa de Kaieski, Grings e Fetter (2015) realizada em duas escolas, uma de idiomas e outra técnica, confirmou o aumento no engajamento, por parte dos alunos, quando os professores passaram a utilizar aplicativos tecnológicos para tirar dúvidas e enviar introduções dos materiais das aulas posteriores. Conforme destacou Mota (2014) "o ato de estudar antes das aulas regulares na escola em nada conflita ou diminui o papel da sala de aula e da presencialidade do professor e dos demais colegas".

Desse modo, pode se apontar como aspectos vantajosos da adoção da tecnologia na educação básica: o aumento na troca de informações, mobilidade e dinâmica entre professor e aluno no processo de aprendizagem. Outra vantagem é o desenvolvimento da autonomia do aluno para buscar conhecimento, e não apenas ser um receptor das informações trazidas em sala de aula pelo professor.

Antes limitados aos muros da escola, o aluno hoje por meio das plataformas tecnológicas os estudantes podem obter acesso a milhões de livros e artigos com uma rápida busca. (BRITO; MATEUS, 2011).

Entretanto, há o outro lado da moeda, quando os dispositivos móveis e aplicativos atrapalham, não apenas em sala de aula, mas em qualquer outro ambiente onde a utilização em excesso ou para outras finalidades cause constrangimentos e desconfortos. Caracterizando desse modo um aspecto negativo da utilização de ferramentas tecnológicas em sala de aula. (BRITO; MATEUS, 2011).

\section{- AGRAVAMENTO DA DESIGUALDADE}

Devido ao contexto de pandemia, a adoção desse novo modelo de ensino é denominado como Ensino Remoto Emergencial, visto que é diferente do ensino a distância, pois até o momento não havia uma regulamentação específica e nem um cronograma definido tal qual acontece com as universidades que possuem aulas a distância.

As aulas remotas, adotadas de forma abrupta, escancarou a desigualdade social que difere os alunos no acesso à educação. Conforme, percebe-se que muitos 
alunos ficaram impedidos de acompanhar as aulas, visto que não possuem acesso à internet e não dispõe de material adequado. Além disto, alguns alunos não possuem um ambiente adequado para estudo, além de falta de acompanhamento e suporte.

Conforme CUNHA, SILVA e SILVA (2020, p. 32) "A Constituição Federal e a Lei de Diretrizes e Bases da Educação preconizam a educação como direito e reforçam o princípio da igualdade de condições para o acesso e permanência na escola". No entanto, compreende-se que a desigualdade social no país, faz com que este direito seja historicamente negado a muitos indivíduos, marginalizando-os de um acesso à educação.

Desse modo, entende-se que o contexto de pandemia se torna um agravante para a desigualdade social e educacional, visto que uma parcela considerável dos alunos enfrentou dificuldade para estudar, pois a necessidade da utilização de ferramentas tecnológicas e acesso à internet são impeditivos para alguns alunos conseguirem participar das aulas.

\section{- CONSIDERAÇÕES FINAIS}

Em virtude dos fatos apresentados, verifica-se que a adoção das inovações tecnológicas fomentou transformações em diferentes esferas da sociedade. Na área da educação, pode-se observar algumas transformações que a tecnologia proporcionou nas relações entre aluno e professor, como também no processo de aprendizagem.

A implementação das inovações tecnológicas na área da educação, assim como em diversas outras áreas, possui aspectos positivos e negativos. Os aspectos

positivos mencionados anteriormente, podem ser comprometidos quando o aluno utiliza de forma incorreta as ferramentas, resultando nos aspectos negativos. Entretanto, não se recomenda a censura dessas ferramentas tecnológicas em sala de aula.

Por fim, conclui-se que os desafios na implementação das inovações tecnológicas na área da educação básica são extensos, e envolve: fortalecer um 
diálogo de todas as partes envolvidas no processo de aprendizagem, construir uma metodologia adequada para auxiliar os professores na adoção de ferramentas tecnológicas e investir em infraestrutura física e nas condições de trabalho para promover os ganhos positivos do uso da tecnologia na educação.

\section{- REFERÊNCIAS BIBLIOGRÁFICAS}

ALENCAR, Gérsica Agripino; PESSOA, Maérico dos Santos; SANTOS, Ana Katarine de F.S.; CARVALHO, Solange R.R.; LIMA, Hommel de A. de B. WhatsApp como ferramenta de apoio ao ensino. São Paulo, 2015. Disponível em: http://brie.org/pub/index.php/wcbie/article/view/6117/4285. Acesso em: 25 de junho de 2021.

AMORIM, M.; BIANCO, P. Material didático em mídia digital: transposição de uma apostila do Colégio Dom Bosco para Tablet computer. 2011. Trabalho de Conclusão do Curso de Design Gráfico, do Setor de Ciências Humanas, Letras e Artes - Universidade Federal do Paraná.

ARAUJO, Sérgio Paulino; VIEIRA, Vanessa Dantas; KLEM, Suelen Cristina dos Santos; KRESCIGLOVA, Silvane Bind. Tecnologia na Educação: Contexto Histórico, Papel e Diversidade. In: JORNADA DE DIDÁTICA, 4, 2017. Anais... 2017

BARRETO, A. de A. A transferência de informação, o desenvolvimento tecnológico e a produção de conhecimento. INFORMARE: Caderno do Programa de Pós-Graduação em Ciência da Informação, Brasília, DF, v. 1, n. 2, p. 2-10, jul./dez. 1995.

Disponível

em:

$\leq$

HYPERLINK

"http://200.20.0.78/repositorios/bitstream/handle/123456789/523/Barreto.pdf?sequen" HYPERLINK

"http://200.20.0.78/repositorios/bitstream/handle/123456789/523/Barreto.pdf?sequen"

HYPERLINK

"http://200.20.0.78/repositorios/bitstream/handle/123456789/523/Barreto.pdf?sequen"

HYPERLINK

"http://200.20.0.78/repositorios/bitstream/handle/123456789/523/Barreto.pdf?sequen" HYPERLINK

"http://200.20.0.78/repositorios/bitstream/handle/123456789/523/Barreto.pdf?sequen" HYPERLINK

"http://200.20.0.78/repositorios/bitstream/handle/123456789/523/Barreto.pdf?sequen" HYPERLINK

"http://200.20.0.78/repositorios/bitstream/handle/123456789/523/Barreto.pdf?sequen" HYPERLINK 
"http://200.20.0.78/repositorios/bitstream/handle/123456789/523/Barreto.pdf?sequen" HYPERLINK

"http://200.20.0.78/repositorios/bitstream/handle/123456789/523/Barreto.pdf?sequen" HYPERLINK

"http://200.20.0.78/repositorios/bitstream/handle/123456789/523/Barreto.pdf?sequen" HYPERLINK

"http://200.20.0.78/repositorios/bitstream/handle/123456789/523/Barreto.pdf?sequen" HYPERLINK

"http://200.20.0.78/repositorios/bitstream/handle/123456789/523/Barreto.pdf?sequen" HYPERLINK

"http://200.20.0.78/repositorios/bitstream/handle/123456789/523/Barreto.pdf?sequen" HYPERLINK

"http://200.20.0.78/repositorios/bitstream/handle/123456789/523/Barreto.pdf?sequen" HYPERLINK

"http://200.20.0.78/repositorios/bitstream/handle/123456789/523/Barreto.pdf?sequen"

http://200.20.0.78/repositorios/bitstream/handle/123456789/523/Barreto.pdf?sequen

ce=1>. Acesso em: 25 de junho de 2021.

BEHAR, Patrícia Alejandra. Modelos pedagógicos em educação a distância. Porto Alegre: Artmed, 2009.

BES, F. D.; KOTLER, P. A Bíblia da Inovação: Princípios fundamentais para levara cultura da inovação contínua às organizações. São Paulo: Leya, 2011.

BRANDÃO, C.R. O que é educação. São Paulo: Editora Brasiliense, 2007.

BRITO, Gláucia da Silva. MATEUS, Marlon de Campos. Celulares, smartphones e tablets na sala de aula: complicações ou contribuições? Curitiba, Paraná. 2011.

Disponível em http://educere.bruc.com.br/arquivo/pdf2011/5943 3667.pdf. Acessoem: 25 de junho de 2021.

CARVALHO, Marly. Inovação: estratégias e comunidades de conhecimento. São Paulo: Atlas, 2009.

CUNHA, Leonardo Ferreira Farias da; SILVA, Alcineia de Souza; SILVA, Aurênio Pereira da. $O$ ensino remoto no Brasil em tempos de pandemia: diálogos acerca da qualidade e do direito e acesso à educação. 2020.

FERRAMENTAS PARA ENSINO REMOTO: Microsoft Teams. O que é o Microsoft Teams? Disponível em: <https://www.ead.ufu.br/mod/book/view.php?id=82948>. Ac

KAIESKI, Naira. GRINGS, Jacques André. FETTER, Shirlei Alexandra. Um estudo sobre as possibilidades pedagógicas de utilização do WhatsApp. Rio Grande do Sul:

Revista

Renote, 2015.

Disponível em: 
https://seer.ufrgs.br/renote/article/view/61411/36314. Acesso em: 29 de junho de 2021.

MOTA, Ronaldo. Inovação e aprendizagem independente na educação básica. Ciência e Natura, v. 36, p. 121-129, 2014.

MOURA, A. M. C. Apropriação do telemóvel como ferramenta de mediação em mobile learning: estudos de caso em contexto educativo. 2011. Disponível em: $\leq$ HYPERLINK "http://repositorium.sdum.uminho.pt/handle/1822/13183" HYPERLINK "http://repositorium.sdum.uminho.pt/handle/1822/13183" "http://repositorium.sdum.uminho.pt/handle/1822/13183" "http://repositorium.sdum.uminho.pt/handle/1822/13183" "http://repositorium.sdum.uminho.pt/handle/1822/13183" "http://repositorium.sdum.uminho.pt/handle/1822/13183" "http://repositorium.sdum.uminho.pt/handle/1822/13183" "http://repositorium.sdum.uminho.pt/handle/1822/13183" "http://repositorium.sdum.uminho.pt/handle/1822/13183" "http://repositorium.sdum.uminho.pt/handle/1822/13183" "http://repositorium.sdum.uminho.pt/handle/1822/13183" "http://repositorium.sdum.uminho.pt/handle/1822/13183" "http://repositorium.sdum.uminho.pt/handle/1822/13183" "http://repositorium.sdum.uminho.pt/handle/1822/13183" HYPERLINK HYPERLINK HYPERLINK HYPERLINK HYPERLINK HYPERLINK HYPERLINK HYPERLINK HYPERLINK HYPERLINK HYPERLINK HYPERLINK HYPERLINK "http://repositorium.sdum.uminho.pt/handle/1822/13183"http://repositorium.sdum.umin ho.pt/handle/1822/13183>. Acesso em: 29 de junho de2021.

PASSOLI, Gabriela. Gestão da inovação e do conhecimento. Curitiba: EnterSaberes 2012.

PINTO, Míriam de Magdala. Tecnologia e inovação. Departamento de Ciências da Administração/UFSC, 2009.

TERRA, José. Inovação: quebrando paradigmas para vencer. São Paulo: Saraiva, 2007.

TORRES, Aline Albuquerque. SILVA, Maria Luiza Rocha da. 0 ambiente Moodle como apoio a educação a distância. Pernambuco, 2008. Disponível em: http://www.aedmoodle.ufpa.br/pluginfile.php/254409/mod forum/attachment/34826 HYPERLINK

"http://www.aedmoodle.ufpa.br/pluginfile.php/254409/mod forum/attachment/34826\% 205/O\%20Ambiente\%20Moodle\%20como\%20ferramenta.pdf" HYPERLINK "http://www.aedmoodle.ufpa.br/pluginfile.php/254409/mod forum/attachment/34826\% 205/O\%20Ambiente\%20Moodle\%20como\%20ferramenta.pdf" HYPERLINK "http://www.aedmoodle.ufpa.br/pluginfile.php/254409/mod forum/attachment/34826\% 205/O\%20Ambiente\%20Moodle\%20como\%20ferramenta.pdf" HYPERLINK "http://www.aedmoodle.ufpa.br/pluginfile.php/254409/mod forum/attachment/34826\% 
205/O\%20Ambiente\%20Moodle\%20como\%20ferramenta.pdf" HYPERLINK "http://www.aedmoodle.ufpa.br/pluginfile.php/254409/mod forum/attachment/34826\% 205/O\%20Ambiente\%20Moodle\%20como\%20ferramenta.pdf" HYPERLINK "http://www.aedmoodle.ufpa.br/pluginfile.php/254409/mod forum/attachment/34826\% 205/O\%20Ambiente\%20Moodle\%20como\%20ferramenta.pdf" HYPERLINK "http://www.aedmoodle.ufpa.br/pluginfile.php/254409/mod forum/attachment/34826\% 205/O\%20Ambiente\%20Moodle\%20como\%20ferramenta.pdf" HYPERLINK "http://www.aedmoodle.ufpa.br/pluginfile.php/254409/mod forum/attachment/34826\%2 05/O\%20Ambiente\%20Moodle\%20como\%20ferramenta.pdf" HYPERLINK "http://www.aedmoodle.ufpa.br/pluginfile.php/254409/mod forum/attachment/34826\%2 05/O\%20Ambiente\%20Moodle\%20como\%20ferramenta.pdf" HYPERLINK "http://www.aedmoodle.ufpa.br/pluginfile.php/254409/mod forum/attachment/34826\%2 05/O\%20Ambiente\%20Moodle\%20como\%20ferramenta.pdf" HYPERLINK "http://www.aedmoodle.ufpa.br/pluginfile.php/254409/mod forum/attachment/34826\%2 05/0\%20Ambiente\%20Moodle\%20como\%20ferramenta.pdf" HYPERLINK "http://www.aedmoodle.ufpa.br/pluginfile.php/254409/mod forum/attachment/34826\%2 05/O\%20Ambiente\%20Moodle\%20como\%20ferramenta.pdf" HYPERLINK "http://www.aedmoodle.ufpa.br/pluginfile.php/254409/mod forum/attachment/34826\%2 05/0\%20Ambiente\%20Moodle\%20como\%20ferramenta.pdf" HYPERLINK "http://www.aedmoodle.ufpa.br/pluginfile.php/254409/mod forum/attachment/34826\%2 05/0\%20Ambiente\%20Moodle\%20como\%20ferramenta.pdf"5/0\%20Ambiente\%20Mo odle\%20como\%20ferramenta.pdf. Acesso em: 29 de junhode 2021. 